\title{
Survey on Energy Efficient Routing Protocols by Clustering and Load balancing in Wireless Sensor Networks
}

\author{
Bharati.S.Shetty ${ }^{1}$, Suresha ${ }^{2}$ \\ ${ }^{1}$ Dept. of Information Technology, Walchand College of Engineering, Sangli, India \\ ${ }^{2}$ Sri Venkateshwara College of Engineering, Vidyanagar, Bengaluru, India \\ *Corresponding Author Email: ${ }^{1}$ Bharathishetty5@gmail.com, ${ }^{2}$ principal@svcengg.com
}

\begin{abstract}
Wireless Sensor Network (WSN) is consisting of nodes which deployed to sense the physical environment. In WSN there is excessive growth in performance evaluation and analysis techniques, because of fast development in Information and Communication Technologies (ICT). The main resource problem in WSN is the energy of sensor nodes. There is need of routing protocol which consumes less energy during communication. A reliable and robust routing protocol which limits the excess energy consumed by nodes during forwarding and receiving messages results in energy efficiency protocol. This survey work provides the advantages, drawbacks and comparison of five energy efficient routing protocols.
\end{abstract}

Keywords: Wireless Sensor Networks (WSNs), Energy Driven Architecture (EDA), Energy efficient routing protocols, Clustering, Load Balancing.

\section{Introduction}

The sensor network is network of scattered sensors nodes which are capable to sense, compute and transmit. Application of Wireless Sensor Network (WSN) is in engineering, health, environment and monitor remote locations with less expense.

The main resource problem in WSN is the energy consumption during communication [1]. Which is one of the criteria affecting the network performance and life time? Since many researches were undergone for minimizing the energy consumption of sensor nodes during transmission [2]. The routing protocols are implemented for optimizing the energy consumption and thereby increasing the performance of the network. The recent research shows that Energy-Driven Architecture (EDA) model is applied in WSN to get energy consumption of node.

EDA as a durable architecture which reflects all principal energy constituents of wireless sensor networks applications [3]. The EDA model recognizes universal and critical energy-consuming components of the network. EDA as a component-based model is applied to position sensor nodes according to energy consumption through their components. By observing this architecture it is possible to optimize and balance energy consumption in WSN.

The vital aim is to yield energy map architecture of a WSN application that covers necessary and definable energy components and the associations among these components which are capable to discover the approaches for minimizing the total energy depletion of the sensor network. In various sensor network applications, prolonging the lifetime of WSN and ensuring packet transfer delays are acute for attaining suitable quality of service (QoS)[4].The energy consumption of sensors is modeled depending on its energy overwhelming components and tasks.

Energy balancing is one of the factor missed during the energy consumption, many routing protocols are implemented lately for the energy consumption.
In wireless sensor network, the energy is the main factor running the network alive, but the consumption of energy by the sensor network is high. The sensor network are generally classified in to sensor nodes, each node consumes larger energy for its processing. Due to the range and time delay of operation the energy consumption is much greater than normal. Various routing protocols are proposed earlier, some of them are cluster based, tree based clustering, multi hop routing etc.

The implementation of routing protocols for optimizing the energy balancing and thereby increasing the performance of the system. To enhance the performance clustering the sensor node is one of the approaches. Clustering using K-means is popular practice in sensor network [5]. One of the advantages of clustering is segregation of larger databases in short period of time.

The energy is utilized by the sensor nodes of wireless network are clustered for the effective balancing of energy [6]. An energy efficient cluster routing protocol optimizes the energy balancing in wireless sensor nodes.

In this survey different energy efficient routing protocols are considered for defines their advantages and drawbacks. This survey provides a proper selection of energy efficient routing protocol for different applications of WSN by considering various attributes.

Multiple sink single hop are implemented to reduce the node distance for the consumption and balancing of energy for processing.

Decentralized hierarchical cluster-based routing protocol makes use of multi-criterion clustering algorithm and a cluster-based routing algorithm. These algorithms are performed con-currently.

Hierarchical Energy Efficient Clustering Algorithm (HEEC) is to enhance the life time sensor network by optimizing high energy consumption and evenly distributing the load among the sensor nodes. Cluster Arrangement Energy Efficient Routing Protocol (CAERP) is to overcome excess energy consumption better than other clustering process in wireless sensor network. 
The Centralized Energy Efficient Distance (CEED) routing protocol Entail distributed cluster head selection algorithm for clustering and balancing energy in sensor nodes.

\section{Energy Efficient Routing Protocol}

This literature survey is discuss the different routing protocols with main objective of balancing energy for improving network life cycle and energy consumption of wireless sensor network.

\section{A. Multiple sink single hope Routing Protocol}

Problem (Limitation of the Past Work): Unstable energy balancing in sensor nodes of single hop sensor network leads to large consumption of energy and lower the network life cycle.

Some of the recent research work is carried to optimize the energy consumption in the single hop senor network, somehow the balancing of energy in the network be a question mark. Jain et al[7] propose multiple sink single hop routing protocol, the sensor node are randomly assigned for deployment. Nodes are connected to sink depending on the distance and transmission energy. If distance between node and sink is less than transmission range, node is get connected to sink. In this way, all nodes are get connected to single or multiple sinks. Some of nodes may not be connected to any sink because they are deployed far away from network which are not in transmission range of any sink.

Network restructuring is done periodically. Energy consumption of each sink node is calculated and high energy consuming sink is detected. Unique node is one which is connected to single sink Such node is traced out in network. Nodes apart from unique nodes, which are connected to other sink are detected. Such a nodes are connected to another sinks by considering energy consumption must not cross the threshold of that sink. Sink which are consumes more energy is reduced in this way. Above process is frequently applied for all sinks, according to their increasing order of energy consumption. At the end of this process, network consumes less energy is formed.

Multiple sink single hops are implemented to reduce the node distance for the consumption and balancing of energy for processing. Thus the energy consumed by each node is reduced and energy balanced for sensor nodes and thereby improving the lifetime of sensor network. In this routing protocol the sink randomly moved at certain position and it is unable to predict the position of sink. Thus this work will be upgraded by deploying the sink at fixed position rather than moving randomly.

Drawback: In Multiple Sink Single Hop Routing, the sink moves random position and it is difficult to find the position of the sink.

\section{B. Decentralized hierarchical cluster-based routing protocol}

Problem (Limitation of the Past Work): A centralized cluster based routing protocol for energy balancing and consumption in sensor nodes. However the energy is deflected for sensor nodes and consumes large amount of energy.

Decentralized hierarchical cluster-based routing protocol makes use of multi-criterion clustering algorithm and a cluster-based routing algorithm. These algorithms are performed con-currently. The multiple set of cluster heads are selected for data aggregation and relay transmission

In multi-criterion clustering algorithm .To select the cluster head it considers local information of each node which contains distance from base station, residual energy and proximity to its neighbor The residual energy is the important aspect for load balancing and energy consumption of cluster head. The distance is calculated based on route created from base station to cluster head. Based on the proximity, intra-cluster and inter cluster energy consumption is managed. Finally, selected cluster head has optimal path to base station.
Minimum spanning tree is constructed which covers the entire network in cluster based routing algorithm to minimize the message transmission cost .Cluster based routing algorithm optimize resource usage and delay the death of sensor nodes.

Sabet et al[8] proposes a decentralized hierarchical cluster based routing algorithm for energy consumption and energy balancing in wireless sensor network. Cluster based routing algorithm was implemented for reduce the energy consumed by each individual sensor nodes. The protocol works in multiple rounds and every rounds has two phases. The first phase is cluster route setup which include another two sub phases. Based on local information of each node, first sub phase create routing tree among the eligible cluster heads. In second sub phase cluster formation is done with suitable cluster head. Data transmission is second phase, in which data is transferred to base station through the established tree.

Drawback: The sensor nodes are placed randomly at near distance of transmission, which leads to consumption of energy largely by near nodes and not balance for the entire clustered node in wireless sensor network.

\section{Hierarchical Energy Efficient Clustering Algorithm (HEEC)}

Problem (Limitation of the Past Work): Direct transmission in sensor nodes leads to unbalanced energy consumed by the senor nodes

The main issue occurs during routing is energy balancing, Rajeshwari et al [9] propose a Hierarchical Energy Efficient Clustering Algorithm (HEEC) for the energy balancing and consumption during the processing of wireless senor network.

The main goal of the HEEC is to enhance the life time sensor network by optimizing high energy consumption and evenly distributing the load among the sensor nodes. To group all sensor nodes, clustering is used in HEEC. The cluster formation is carried out after selecting the cluster head by base station.

The selected cluster head creates routing tree for every destination node. Destination-Sequenced Distance-Vector Routing protocol is used to find optimal path from source to destination. After analyzing the optimal path which consumes minimum energy and less communication delay, cluster head selects that path for data transfer.

For re-electing the cluster head the base station analyzes the energy level of sensor nodes in each round. Base station also verify previous cluster head whether it has enough energy to transfer the data packet. If node not have enough energy ,base station again analyzes the energy level, aliveness and distance of sensor nodes. Based on this high energy node is elected and announced as new cluster head.

Data loss and data fusion problems are eliminated by re-electing the cluster head periodically. The methods like clustering, reelecting cluster head and construction of routing tree are used to transfer data packets securely in WSN.

The protocol incorporates clustering algorithm to create effective cluster of sensor node for handling the energy consumed by each sensor node. This protocol finds a least distance of transmission and consumes much energy and balance the energy between nodes. Thus the HEEC protocol forms better in improving the consumption and balancing energy in wireless sensor network.

Drawback: Energy is utilized by only the nodes and cluster heads which are in transmission and energy is balanced and consumed only for the particular nodes and remains are unstable.

\section{Cluster Arrangement Energy Efficient Routing Protocol (CAERP)}

Problem (Limitation of the Past Work): The recent research proposes Q-LEACH (quadrant based low energy adaptive cluster hierarchy) protocol for energy consumption, but the energy is not stable and leads to unstable operational state for larger networks. 
Depending on different applications, clustering arrangements are classified into homogeneous and heterogeneous, static and dynamic, centralized and distributed. Clustering has many advantages in terms of energy conservation, data aggregation and load balancing in network.

Cluster Arrangement Energy Efficient Routing Protocol (CAERP) is proposed by Vijayan et al[10] to balance and consume less energy than Q-LEACH protocol. This protocol also focused to overcome excess energy consumption better than other clustering process in wireless sensor network.

CAERP works in four stages, clustering, cluster head selection, routing and data transmission. Initially network is divided into clusters with different sizes. Energy is conserved during data transmission as the cluster is near to base station. Clusters near to base stations have small in size and clusters far from base stations have large in size. The overall system performance has to enhance and reduces maintenances of network to distribute energy dissipated.

Researches are carried out to optimize the energy consumption in wireless sensor network. Q-Leach is one of the recently proposed method for energy consumption, however the protocol are not efficient for energy balancing. In data transmission the energy is unbalanced for sensor nodes and it is not appropriate protocol for applying large networks. The energy consumed by the sensor node differs from one other. The CAERP protocol utilizes clustering and distributed multi-hop routing for efficient cluster formation and balance the energy in node during routing. The clustering uses centralized method and therefore the cluster requires less energy for transmission. Thus the CAERP is an efficient method for energy consumption and energy balancing in sensor network.

Drawback: Clustering techniques are proposed earlier and efficiency of the output is out of date with the recently proposed methods. The methodology will be upgraded by proposing innovative routing for overcome energy efficiency and other factors like QoS, security etc.

\section{E. Centralized Energy Efficient Distance (CEED) Based Routing Protocol}

Problem (Limitation of the Past Work): Many hierarchical clusters based routing protocol is proposed for energy consumption and balancing. The efficiency of the recent methodology lacks at some point and balancing is one of the factor affecting the efficiency of the wireless sensor network.

The Centralized Energy Efficient Distance (CEED) routing protocol is proposed by Gawade et al [11] to overcome the energy balancing in wireless sensor network. This method consists of distributed cluster head selection algorithm for clustering and balancing energy in sensor nodes.

The minimum no. of cluster are calculated by considering energy dissipated in overall network during a single round cluster head selection, cluster formation and steady state phases.

Cluster head selection is based on residual energy, selection probability and distance of node to base station which improves threshold value. Elected cluster head broadcasts the message which contains Id, header with control message and spreading code to eliminate the inter-cluster interference.

Main problem to assign a specific cluster head to every node, once cluster head is selected. Energy consumption should be balanced around the cluster head during cluster formation.

To reduce inter-cluster interference and energy consumption TDMA schedule is applied by allowing node to turn off the radio components. Every non cluster node receives TDMA schedule before completion of cluster formation.

According to TDMA schedule, every non-cluster head node sends data to its cluster head in steady state phase. Every cluster communicates through unique spreading code to avoid intercluster interference. Cluster head aggregates data to form single packet, after receiving the data from cluster member.

Then cluster head forward these packets to base station. High energy consumed by cluster head which are away from base station. To achieve energy balancing, data will be transmitted from multiple hopes towards base station.

However the balancing of energy in sensor node is most influencing factor affecting the performance of wireless sensor network. The CEED protocol find optimal cluster and ranges of node from the cluster head for consuming less energy and transmission of data in the sensor network and each sensor node energy is balanced for effective transmission. This protocol extended by implementing multi hop routing protocol for effective energy consumption and balancing for processing multiple wireless senor networks.

Drawback: However the efficiency of proposed protocol is not efficient and work is extended by proposing multi hop routing protocol for enhancing energy balancing and energy consumption in wireless sensor network.

\section{Comparison of Protocols}

Table 1: Comparison of various Energy efficient routing protocols

\begin{tabular}{|c|c|c|c|c|c|c|}
\hline $\begin{array}{c}\text { Energy efficient routing } \\
\text { protocols }\end{array}$ & Hops & Sinks & $\begin{array}{c}\text { Mobility of } \\
\text { Sink }\end{array}$ & Topology & $\begin{array}{c}\text { Centralized/ } \\
\text { Decentralized }\end{array}$ & Sink position \\
\hline $\begin{array}{c}\text { Multiple sink single hope } \\
\text { Routing Protocol }\end{array}$ & Single & Multiple & Yes & Random & Decentralized & Variable \\
\hline $\begin{array}{c}\text { Decentralized hierarchical } \\
\text { cluster-based routing protocol }\end{array}$ & Multiple & Single & No & Clustering & Decentralized & Fixed \\
\hline HEEC & Single & Single & No & Clustering & Centralized & Fixed \\
\hline CAERP & Multiple & Single & No & Clustering & Centralized & Fixed \\
\hline CEED & Multiple & Single & No & Clustering & Centralized & Fixed \\
\hline
\end{tabular}

Energy efficient routing protocols in which different parameters are considered to compare the performance of the protocols. The parameters considered are no. of hops, no. of sinks, network topology, centralized or decentralized and sink position.

According to this survey, multiple sink single hope routing protocol and HEEC which have single hop and other protocols like Decentralized hierarchical cluster based routing protocol, HEEC, CAERP and CEED have multiple hops. Multiple sink single hope routing protocol have multiple sinks whereas decentralized hierarchical cluster based routing protocol, HEEC, CAERP and CEED have single sink. Decentralized hierarchical cluster based routing protocol supports mobility of sink where as other protocols doesn't support mobility of sink. The topology of multiple sink single hope routing protocol is random however the other protocols use clustering. Multiple sink single hope routing protocol and decentralized hierarchical cluster based routing protocol are decentralized. The HEEC, CAERP and CEED are centralized. The sink position of HEEC, CAERP, CEED and decentralized hierarchical cluster based routing protocol is fixed. The sink position of multiple sink single hope routing protocol is variable.

Based on this analysis and according to need of specific application, able to select particular protocol. The survey helps researches to develop more energy efficient routing protocols. 


\section{Conclusion}

In this survey limitations of past work for each protocol is considered, based on that recent energy efficient and load balancing routing protocol are discussed with their advantages and drawbacks. The protocols discussed are Multiple sink single hope routing protocol, Decentralized hierarchical cluster based routing protocol, HEEC, CAERP, CEED. The comparison of energy efficient routing protocols in WSN is done by various parameters. The parameters considered are no. of hops, no. of sinks, network topology, centralized or decentralized and sink position.

\section{References}

[1] Kim, Hye- Young. "An energy-efficient load balancing scheme to extend lifetime in wireless sensor networks", Cluster Computing, Vol. 19, No. 1, pp: 279-283, 2016.

[2] Nguyen, Khanh-Van, et al. "An energy efficient and load balanced distributed routing scheme for wireless sensor networks with holes." Journal of Systems and Software, Vol. 123, No. 1, pp.: 92105, 2017.

[3] Doan B. Hoang, Najmeh Kamyabpour, "An Energy Driven Architecture for Wireless Sensor Networks." in International Conference on Parallel and Distributed Computing, Applications and Technologies, 2012.

[4] Jiang, Dingde, Wenpan Li, and Haibin Lv. "An energy-efficient cooperative multicast routing in multi-hop wireless networks for smart medical applications." Neurocomputing, Vol. 220, No. 1, pp.: 160-169, 2017.

[5] Lu, Yuting, Yongquan Zhou, and Xiuli Wu. "A Hybrid Lightning Search Algorithm-Simplex Method for Global Optimization." Discrete Dynamics in Nature and Society, Vol. 1, No. 1, 2017.

[6] Kaur, Supreet, and Rajiv Mahajan. "Hybrid meta-heuristic optimization based energy efficient protocol for wireless sensor networks." Egyptian Informatics Journal, Vol. 1, No. 1, 2018.

[7] Jain, Tapan Kumar, Davinder Singh Saini, and Sunil VidyaBhooshan. "Lifetime optimization of a multiple sink wireless sensor network through energy balancing." Journal of Sensors, Vol. 1, No.1, 2015

[8] Sabet, Maryam, and Hamid Reza Naji. "A decentralized energy efficient hierarchical cluster-based routing algorithm for wireless sensor networks" AEU-International Journal of Electronics and Communications, Vol. 69, No. 5, pp: 790-799, 2015

[9] Rajeshwari, P., B. Shanthini, and Mini Prince. "Hierarchical energy efficient clustering algorithm for WSN" Middle East Journal of Scientific Research, Vol. 23, No. 1, pp: 108-117, 2015

[10] Vijayan, K., and ArunRaaza. "A novel cluster arrangement energy efficient routing protocol for wireless sensor networks." Indian Journal of science and Technology, Vol. 9, No.2, 2016

[11] Gawade, Rohit D., and S. L. Nalbalwar. "A centralized energy efficient distance based routing protocol for wireless sensor networks", Journal of Sensors, Vol. 1, No. 1, 2016. 